\title{
With the Tsunami of Immunotherapy, Will Soft Tissue Sarcoma Remain an Immune Desert?
}

\section{Georges El Hachem*, Maroun Sadek and Spyridon Sideris}

Department of Medical Oncology, Jules Bordet Institute, Brussels, Belgium

"Corresponding author: El Hachem Georges, MD, Department of Medical Oncology, Jules Bordet Institute, 121 Boulevard de Waterloo, Brussels 1000, Belgium, Tel: +32485853628, +3225413766; E-mail: georges.elhachem@bordet.be, george.el.hashem@hotmail.com

Received date: January 09, 2018; Accepted date: January 16, 2018; Published date: January 23, 2018

Copyright: (c) 2018 El Hachem G, et al. This is an open-access article distributed under the terms of the Creative Commons Attribution License, which permits unrestricted use, distribution, and reproduction in any medium, provided the original author and source are credited.

\begin{abstract}
Soft tissue sarcomas (STSs) are rare, aggressive and heterogeneous malignancies with poor outcomes. They include more than 50 different histologic subtypes with variable molecular and cytogenetic profiles responsible for the heterogeneity of these tumors. Radical surgery, chemotherapy and radiation therapy are still the mainstay of treatment with limited therapeutic options in case of metastatic and locally advanced unresectable disease. The current available cytotoxic and targeted therapies are only offering short living disease control. In the era of immunotherapy, STSs were affected by this 'tsunami' with very promising results, but larger randomized trials are still needed to prove their benefit in terms of survival and efficacy. They are not included yet in the standard of care. In this review of the literature, we are developing the active immunotherapeutic strategies (vaccines), as well as the passive strategies (adoptive cell therapy) and the checkpoint inhibitors emphasizing on the most recent results and on the limitation of the immunotherapy in this heterogenic media.
\end{abstract}

\section{Rationale for Immunotherapy in Soft Tissue Sarcomas}

Sarcomas are a heterogeneous group of tumors with wide expression of immunogenic proteins and antigens, including the cancer-testes antigen family (NY-ESO-1, MAGE-A3, PRAME and LAGE-1), gangliosides (GM2, GD2 and GD3), sarcoma specific-fusion proteins (SSX, FOXO1, EWSR1 and TLS CHOP) and heat shock proteins [1]. These proteins represent attractive potential targets in STS. The earliest evidence of immune reactivity was seen in sarcoma tumors when William B. Coley demonstrated how the patients were able to clear distant sarcomatous metastatic lesions by injecting other tumors with a live streptococcal preparation called "Coley's toxin". Due to its unpredictable toxicity profile and the heterogeneous mode of preparation, this essay of immune reaction remained a historic pioneering work [20]. Sarcomas are rare tumors with many histologic subtypes, leading to a rate limiting step in the development of immune therapy in this field. Additionally, the mesenchymal media and the predominant fibrous tissue are responsible for some immune resistance of these tumors. However, many data for immunotherapy are accumulating including the use of vaccines, adoptive immune therapy and checkpoint inhibitors.

\section{Vaccines}

Vaccine therapy has been the most investigated immunotherapy modality in soft tissue sarcomas given the low toxicity rate in an individualized nature of therapy. Given the diversity of tumor types and the wide expression of specific epitopes, STSs became ideal vaccine targets. The therapeutic effect of cancer vaccines depends mainly on the dendritic cells activation in the presence of immune antigens [3]. Initial studies showed inefficiency of the vaccine therapy because the vaccines were made from entire tumor cells [4]. Then, vaccines were based on an epitope generated from a fusion peptide, SYT-SSX, found in $85-90 \%$ of STS without demonstrating any objective response $[5,6]$.
Another personalized peptide vaccine was created by Takahashi and colleagues with multiple tumor antigens chosen according to preexisting peptide-specific IgG titers. They demonstrated an overall survival (OS) of 9.6 months associated with disease stabilization in $30 \%$ of the patients. [7]. Moreover, Neeta Somaiah and colleagues presented an encouraging phase I study (the C131 phase I trial) enrolling 25 patients, previously treated for recurrent or metastatic STS and being positive for the tumor antigen NY-ESO-1. Among these patients, the histologies were synovial sarcoma (14 cases), myxoid/ round cell liposarcoma (MRCL) (9 cases) and other subtypes (2 cases). They were treated with an active immunotherapy regimen CMB305 that generates and expands T cells against NY-ESO-1. CMB305 has two components: LV305, a dendritic cell-targeting lentiviral vector encoding full-length NY-ESO-1 RNA, and G305, a potent TLR-4 agonist which is co-administered with the full-length protein in a sequential protocol. At data cut-off, the median OS was not reached. The 12 and 18 -month OS rates were $83.1 \%$ and $76.2 \%$, respectively. This regimen was able to control tumor growth with $64 \%$ of the patients achieving a stable disease. However, there was no reported objective response [8].

\section{Immune Checkpoint Blockade}

Immunologic checkpoint blockade with antibodies that target cytotoxic $\mathrm{T}$ lymphocyte-associated antigen 4 (CTLA-4) and the programmed cell death protein 1 pathway (PD-1/PD-L1) have been promising in a variety of malignancies, and are currently a standard of care in many types of tumors.

Some observations based on trials of immune checkpoint blockade suggest that this strategy might be useful and applicable for soft tissue sarcomas. Kim et al. recently characterized the expression of PD-1 and PD-L1 across several STS subtypes. 105 cases of STS were analyzed by immunohistochemistry. The largest represented subtypes were leiomyosarcoma (LMS), synovial sarcoma, undifferentiated sarcoma 
and myxoid liposarcoma. For all STS combined, intratumoral infiltration of PD-1 positive lymphocytes was seen in $65 \%$ of cases and PD-L1 tumor expression was seen in $58 \%$. It was also noticed that $100 \%$ of undifferentiated sarcomas had positive expression of PD-1, compared to $10 \%$ in myxoid liposarcoma. These data suggest that certain STS subtypes may be more amenable to immune checkpoint blockade using anti-PD-1 or anti-PD-L1 [9].

In the American Society of Clinical Oncology (ASCO) 2017 meeting, the results of the phase II Alliance A091401 trial were presented. It included 42 patients who were treated with the duo Nivolumab $3 \mathrm{mg} / \mathrm{kg}$ plus Ipilimumab $1 \mathrm{mg} / \mathrm{kg}$, given every 3 weeks for four cycles, after which they continued with Nivolumab monotherapy until unacceptable toxicity, disease progression, or up to 2 years. 38 patients were evaluable: the overall response rate was $16 \%$ with one patient with myxofibrosarcoma and another one with uterine leiomyosarcoma reaching a complete response. Additionally, five partial responses were seen: three patients with undifferentiated pleomorphic sarcoma/malignant fibrous histiocytoma (UPS/MFH) and one each with non-uterine LMS and angiosarcoma. The OS was a median of 14.3 months, with a 12 months OS rate of $54 \%$ at the time of analysis. These survival results exceeded the expectations for metastatic sarcoma patients. Thus, expansion studies in patients with liposarcoma (LPS) and UPS/MFH are under their ways of development. In the same trial, another 43 patients were given nivolumab $3 \mathrm{mg} / \mathrm{kg}$ every 2 weeks with the same stopping criteria as for the combination. The response rate among these participants was lower: $5 \%$ of 38 evaluable patients. The median OS was 10.7 months and $40 \%$ were alive at the 12 months time-point [10].

In a similar way, Pembrolizumab showed a potential therapeutic option in a sub-group of sarcoma patients. Tawbi et al. underwent a phase II trial, the SARC028, which included 84 patients diagnosed with locally advanced uresectable or metastatic soft tissue sarcoma (42 patients) and bone sarcoma (42 patients). Patients had received up to 3 previous lines of cytotoxic treatments. They were treated with Pembrolizumab at a dose of $200 \mathrm{mg}$ every 3 weeks. Responses were observed in $18 \%$ of patients with soft-tissue sarcoma, as well as in $5 \%$ of patients with bone sarcoma. One case of UPS achieved a complete response and six were partial responses: three patients with UPS, two with LPS and one with synovial sarcoma. There were no responses seen neither in the LMS subgroup (10 patients) nor in the Ewing sarcoma cases (13 patients). The median duration of response was 33 weeks, while the longest response lasted for 86 weeks [11].

The role of PDL1 as a predictive or prognostic biomarker in STS remains debatable [12]. There is also a randomized, open label, phase II trial of CMB305 in combination with Atezolizumab in synovial sarcoma and MRCL underway. (IMDZ-C232; NCT02609984). Additionally, other studies, exploring other immune checkpoint inhibitors targeting other pathways (TIM3, BTLA, 4-IBB and LAG-3) may add a beneficial value to the treatment of STS.

\section{Adoptive Immunotherapy}

Adoptive cellular immune therapy consists of infusing immunemanipulated T-cells into a tumor in order to evoke an antitumor response. The demonstration of effective adoptive therapy in sarcoma patients was carried out in a study using autologous $\mathrm{T}$ cells engineered with T-cell receptor directed at NY-ESO-1 antigen in synovial sarcoma patients where $80 \%$ of the cases express this antigen. Its expression was an inclusion criterion for this study. Patients were initially lympho- depleted using Cyclophosphamide and Fludarabine. Then, autologous $\mathrm{T}$ cells genetically engineered to recognize NY-ESO-1 and HLA$A^{\star} 0201$ antigens were infused. Partial responses occurred in 4 of 6 sarcoma patients, with one response reaching 18 months duration. This therapy was well tolerated with no off-target effects [13]. Nontheless, the response of the adoptive T-cell therapies remains HLA restricted which limits the proportion of patients that can be adequately treated.

Genetically engineered NY-ESO-1 T cells are being evaluated in 2 ongoing trials for patients with metastatic synovial sarcoma (NCT01343043 and NCT03250325). Besides, the role of chimeric antigen receptor CAR-T cell targeting the GD2 protein in children and young adults with sarcomas and rhabdomyosarcomas is also under evaluation in a phase I trial (NCT00743496).

\section{Future Perspectives}

Despite all the progress of the immunotherapy and the fact it is a standard of care in many tumors, it has not yet matured enough to reach strong indications in soft tissue sarcoma. Knowing that these tumors harbor many genetic mutations and translocations, they may serve as a potent immune active media. The researchers and immunologists are trying to overcome the resistance to immunotherapy by transforming the immune desert into an inflamed media. One option is to work on modifying or up-regulating the tumor antigens leading to an increased immunogenicity. These epigenetic changes can be obtained after the exposure of the sarcoma cells to demethylating agents: Decitabine lead to an increase in the tumor antigen expression, resulting in further activation of $\mathrm{T}$ cell immunity. [14]. In the same perspective, Pollack et al. showed an upregulation of cancer-testes antigen expression with the use of Decitabine in chondrosarcoma cell lines. Likewise, the histone deacetylase (HDAC) inhibitors were tested in vitro with promising responses [15].

Another way to boost the immune activation is the innovative approach of combining radiation therapy of certain lesions with the immune therapy. This is called the 'abscopal effect' that was tested in melanoma and there are ongoing trials in order to evaluate its efficacy in STS.

Lastly, researchers are trying to identify unique target antigens. They are genetically engineering $\mathrm{T}$ cell receptors to be used for insertion in the patients' lymphocytes. This approach was possible after the evolvement of the tumor cell whole exome sequencing (WES). Future trials are working on this individualized/personalized treatment in heterogeneous tumors like soft tissue sarcoma [16,17] Consequently, not only are the antigen and tumor peptides modified, but there are new approaches for manufacturing genetically programmed activated T cells.

\section{Conclusion}

Soft tissue sarcomas remain one of the most aggressive tumors with high potential to recur and metastasize. Radical surgical treatment, chemotherapy, targeted therapies and radiotherapy are the current standard of care for soft tissue sarcoma. All the current available therapies serve only to prolong survival, trying to improve the quality of life at the expense of much toxicity. Unfortunately, immune therapy is not among the cornerstone therapeutic options. After being a pioneered tumor since 100 years, the application of the immunotherapy in STS remained passive until recently. The cold immune media of sarcoma is being successfully transformed into a hot 
Citation: El Hachem G, Sadek M, Sideris S (2018) With the Tsunami of Immunotherapy, Will Soft Tissue Sarcoma Remain an Immune Desert?. J

Page 3 of 3

active immune ground: discovery of new tumor antigens and further understanding of the mechanisms of $\mathrm{T}$ cell activation. Anti-tumor activity against sarcoma is a reality with many promising results. The main goal of the ongoing trials is to reach with immune therapy long term responses, better disease control and improvement of the patients' outcome.

\section{References}

1. Wilky BA, Goldberg JM (2014) Immunotherapy in sarcoma: A new frontier. Discov Med 17: 201-206.

2. McCarthy EF (2006) The toxins of William B. Coley and the treatment of bone and soft-tissue sarcomas. Iowa Orthop J 26: 154-158.

3. Mahvi DM, Shi FS, Yang NS, Weber S, Hank J, et al. (2002) Immunization by particle-mediated transfer of the granulocyte-macrophage colonystimulating factor gene into autologous tumor cells in melanoma or sarcoma patients: Report of a phase I/IB study. Hum Gene Ther 13: 1711-1721.

4. Dillman R, Barth N, Selvan S, Beutel L, de Leon C, et al. (2004) Phase I/II trial of autologous tumor cell line-derived vaccines for recurrent or metastatic sarcomas. Cancer Biother Radiopharm 19: 581-588.

5. Kawaguchi S, Wada T, Ida K, Sato Y, Nagoya S, et al. (2005) Phase I vaccination trial of SYT-SSX junction peptide in patients with disseminated synovial sarcoma. J Transl Med 3: 1.

6. Sato Y, Nabeta Y, Tsukahara T, Hirohashi Y, Syunsui R, et al. (2002) Detection and induction of CTLs specific for SYT-SSX-derived peptides in HLA-A24(+) patients with synovial sarcoma. J Immunol 169: 1611-1618.

7. Takahashi R, Ishibashi Y, Hiraoka K, Matsueda S, Kawano K, et al. (2013) Phase II study of personalized peptide vaccination for refractory bone and soft tissue sarcoma patients. Cancer Sci 104: 1285-1294.

8. Somaiah N, Chawla SP, Block MS, Morris JC, Do KT, et al. (2017) Immune response, safety, and survival impact from CMB305 in NYESO-1+ recurrent soft tissue sarcomas (STS). J Clin Oncol 35: 11006.
9. Kim JR, Moon YJ, Kwon KS, Bae JS, Wagle S, et al. (2013) Tumor infiltrating PD1-positive lymphocytes and the expression of PD-L1 predict poor prognosis of soft tissue sarcomas. PLoS One 8: e82870.

10. D'Angelo SP, Mahoney MR, Tine BAV, Atkins JN, Milhem MM, et al. (2017) A multi-center phase II study of nivolumab +/- ipilimumab for patients with metastatic sarcoma (Alliance A091401). J Clin Oncol 35: 11007.

11. Tawbi HA, Burgess M, Bolejack V, Van Tine BA, Schuetze SM, et al. (2017) Pembrolizumab in advanced soft-tissue sarcoma and bone sarcoma (SARC028): A multicentre, two-cohort, single-arm, open-label, phase 2 trial. Lancet Oncol 18: 1493-1501.

12. Kim JR, Moon YJ, Kwon KS, Bae JS, Wagle S et al. (2013) Tumor infiltrating D1-positive lymphocytes and the expression of PD-L1 predict poor prognosis of soft tissue sarcomas. PLoS One 8: e82870.

13. Robbins PF, Morgan RA, Feldman SA, Yang JC, Sherry RM, et al. (2011) Tumor regression in patients with metastatic synovial cell sarcoma and melanoma using genetically engineered lymphocytes reactive with NYESO-1. J Clin Oncol 29: 917-924.

14. Krishnadas DK, Bao L, Bai F, Chencheri SC, Lucas K (2014) Decitabine facilitates immune recognition of sarcoma cells by upregulating CT antigens, MHC molecules, and ICAM-1. Tumour Biol 35: 5753-5762.

15. Pollack SM, Li Y, Blaisdell MJ, Farrar EA, Chou J, et al. (2012) NYESO-1/ LAGE-1s and prame are targets for antigen specific $t$ cells in chondrosarcoma following treatment with 5-Aza-2-deoxycitabine. PLoS One 7: e32165.

16. Lu YC, Yao X, Crystal JS, Li YF, El-Gamil M, et al. (2014) Efficient identification of mutated cancer antigens recognized by $\mathrm{T}$ cells associated with durable tumor regressions. Clin Cancer Res 20: 3401-3410.

17. Gros A, Parkhurst MR, Tran E, Pasetto A, Robbins PF et al. (2016) Prospective identification of neoantigen-specific lymphocytes in the peripheral blood of melanoma patients. Nat Med 22: 433-438. 